\title{
Editorial
}

Psychotherapeut 2015 $\cdot 60: 475-476$

DOI 10.1007/s00278-015-0065-0

Online publiziert: 20. Oktober 2015

(c) Springer-Verlag Berlin Heidelberg 2015

\author{
Alexandra Martin ${ }^{1} \cdot$ Jennifer Svaldi ${ }^{2}$ \\ ${ }^{1}$ Klinische Psychologie und Psychotherapie, Bergische Universität Wuppertal, Wuppertal, Deutschland \\ ${ }^{2}$ Klinische Psychologie und Psychotherapie, Eberhard Karls Universität, Tübingen, Deutschland
}

\section{Körperbild und Körperbildstörungen}

Unter „Körperbild“ können alle psychischen Anteile der Körperrepräsentanz verstanden werden. Typischerweise werden verschiedene Dimensionen des subjektiven Erlebens des eigenen Körpers hierunter gefasst wie Wahrnehmung, Kognition, Affekt, aber auch Verhalten. Dazu gehören die Wahrnehmungen des eigenen Körpers oder einzelner Merkmale (visuell-exterozeptiv), aber auch innerer Körpervorgänge und das Körpergefühl (somatosensorischinterozeptiv). Unter den Kognitionen fallen evaluative Aspekte wie die Zufriedenheit mit dem Aussehen oder die Bedeutung des Aussehens für Selbstwert oder Lebensführung. Eng assoziiert damit sind das affektive Erleben und Befinden sowie Auswirkungen auf das Verhalten. „Körperbildstörungen "können in einer oder mehreren der Körperbilddimensionen bestehen. Beispielsweise kann eine bestehende Unzufriedenheit mit dem eigenen Körper einhergehen mit der fehlerhaften Wahrnehmung der äußerlich beobachtbaren Erscheinung oder auch der Vermeidung von sozialen Situationen aufgrund des Aussehens. Wird von Körperbildstörungen im Sinne von psychischen Störungen gesprochen, assoziiert man hiermit v. a. die Essstörungen oder auch die körperdysmorphe Störung.

Die Beiträge dieses Schwerpunkthefts befassen sich mit Besonderheiten des Körperbilds, ihren Mechanismen, ihren Auswirkungen und ihrer Behandlung bei diesen Störungsgruppen und weiteren Personengruppen - wie beispielsweise bei Bestehen sichtbarer Auffälligkeiten im Erscheinungsbild oder bei verschiedenen anderen psychischen Störungen - ebenso wie mit Wünschen und Formen zur Veränderung des äußeren Erscheinungsbilds in der Allgemeinbevölkerung.

Die Arbeit von Appel et al. zeigt auf, dass Piercings und Tattoos beliebte und verbreitete Formen des „Körperstylings“ bei Jugendlichen und jungen Erwachsenen darstellen. Hier zeigt sich eine positive Beziehung zwischen diesen Varianten der Einflussnahme auf das körperliche Erscheinungsbild und der eigenen Attraktivitätswahrnehmung. Es zeigt sich ebenso, dass in der Gruppe mit diesen Körpergestaltungen auch andere aussehensbezogene Verhaltensweisen stärker ausgeprägt sind und insbesondere die Bereitschaft erhöht ist, andere kosmetisch-chirurgische Maßnahmen durchführen zu lassen. Diskutiert wird hier die insgesamt zu beobachtende Offenheit in der Gesellschaft, durch invasive Korrekturen („Schönheitsoperationen") Aspekte des eigenen Aussehens zu verbessern.

In der Übersichtsarbeit von Cordes et al. wird ersichtlich, dass die aufmerksamkeitsbezogene Informationsverarbeitung an der Aufrechterhaltung der Körperunzufriedenheit von Frauen mit (subklinischer) Essstörung und körperunzufriedenen Männern beteiligt zu sein scheint. Diese Erkenntnis dürfte mittelfristig wichtige Implikationen für die Behandlung von Körperbildstörungen haben, indem die derzeitigen psychotherapeutischen Interventionen gezielter auf die Veränderung der aufmerksamkeitsbezogenen Informationsverarbeitung bei der Körperbildbetrachtung ausgerichtet werden können. Wenn auch erste Befunde hinsichtlich solcher Ansätze (z. B. dem Aufmerksamkeitsmodifikationstraining) vielversprechend sind, so zeigt sich auch, dass die Befundlage derzeit noch zu heterogen ist und künftige Studien einen besseren Aufschluss darüber liefern müssen, welche Komponenten der Aufmerksamkeit (z. B. der Abbau einer verstärkten Aufmerksamkeitszuwendung oder einer verstärkten aufmerksamkeitsbezogenen
Vermeidung) unter welchen Bedingungen für eine Reduktion der Körperunzufriedenheit modifiziert werden müssen.

Zwei empirische Beiträge im vorliegenden Themenheft beschäftigen sich mit der Relevanz der Körperunzufriedenheit bei körperunzufriedenen präadoleszenten Mädchen (Schäfer et al.) sowie übergewichtigen und adipösen Kindern und Jugendlichen vor und nach einer stationären Adipositasbehandlung (Hoffmann et al.). Die prospektive Studie von Hoffmann et al. liefert Hinweise, dass eine Unterschätzung (im Vergleich zu einer realistischen Einschätzung) der eigenen Körperform mit einer stärkeren Gewichtsreduktion assoziiert ist. Künftige Studien sollten mögliche Moderatoren dieses $\mathrm{Zu}$ sammenhangs untersuchen. Denkbar ist, dass eine realistischere Einschätzung des eigenen Körpers bei Kindern mit Übergewicht und Adipositas zu vermehrtem Stress und einer stärkeren Nahrungsrestriktion führen, beides Faktoren, die mit einer erhöhten Kalorienaufnahme bei Kindern in Verbindung gebracht wurden (Balantekin und Roemmich 2012; Roemmich et al. 2011; Roemmich et al. 2002). In diesem Zusammenhang liefert die Studie von Schäfer et al. interessante Erkenntnisse dahingehend, dass negative körperbezogene Affekte bei präadoleszenten Mädchen durch die Anwendung instruierter kognitiver Neubewertung signifikant reduziert werden können. Gerade aufgrund der weit verbreiteten Körperunzufriedenheit unter Kindern und Jugendlichen (Berger et al. 2005) und des damit assoziierten erhöhten Risikos für die Entwicklung von Essstörungen (Stice et al. 2011) stellt sich die Frage, inwiefern eine funktionale Regulation negativer körperbezogener Affekte bei Hochrisikogruppen wirksam ist bzw. Einfluss auf die Körper- 
bildstörung bei Patienten mit Essstörung hat. Während es im Erwachsenenbereich präklinische (Wade et al. 2009) und klinische (Naumann et al. under review) Studien gibt, die auf eine kausale Beziehung von Emotionsregulation und Körper(un) zufriedenheit hindeuten, stehen entsprechende longitudinale und experimentelle Untersuchungen mit geeigneten Emotionsregulationskontrollbedingungen im Kindes- und Jugendalter noch aus.

Auch chronische Erkrankungen können mit Veränderungen des Körpererlebens und des Körperbilds einhergehen. Besonders angeborene oder durch Krankheiten und Verletzungen erworbene äußerlich sichtbare Veränderungen wie beispielsweise bei Hauterkrankungen oder nach Mastektomie bei Brustkrebs können stigmatisierend erlebt werden, die Anpassung an die Veränderung erschweren und die Lebensqualität stark beeinträchtigen. Die systematische Befundintegration von Schroeder und Martin zeigt, dass bei jungen Betroffenen mit kongenitaler Brustwanddeformität eine ausgeprägte Unzufriedenheit mit dem Aussehen besteht, damit einhergehend der Selbstwert vergleichsweise geringer ausfallen kann und gerade soziale Interaktionen mit Gleichaltrigen aufgrund von Unsicherheit eingeschränkt werden. Chirurgische Korrekturmaßnehmen werden nicht nur wegen der körperlichen Funktionseinschränkungen, sondern auch aus der Hoffnung aufgesucht, das eigene Aussehen zu „normalisieren“. Tatsächlich weisen Verlaufserhebungen auf eine mehrheitliche Zufriedenheit mit dem Operationsergebnis. Da jedoch nicht bei allen Betroffenen eine (nachhaltige) Verbesserung der Köperakzeptanz resultiert, empfehlen Schroeder und Martin, Körperbildaspekte in die Routinediagnostik bei dieser Zielgruppe aufzunehmen. Hierdurch kann frühzeitig überprüft werden, ob und welche Zusatzmaßnahmen zur Veränderung der Körperakzeptanz, zum Abbau sozialer Ängste oder Selbstwertstärkung - ähnlich wie bei anderen Personengruppen mit belastend erlebten sichtbaren Auffälligkeiten - zum Einsatz kommen sollten.

In 2 Beiträgen dieses Hefts der Zeitschrift Psychotherapeut steht die Psychotherapie von Körperbildstörungen im Fokus. Dyer et al. untersuchen im naturalis- tischen Setting ambulanter Psychotherapie, wie körperbildspezifische Therapieelemente der kognitiven Verhaltenstherapie genutzt werden. Zu den körperbildspezifischen Interventionen zählen Maßnahmen zur Veränderung negativer körperbezogener Kognitionen, Abbau von Vermeidungs- und Kontrollverhalten ebenso wie körperorientierte Techniken zum Aufbau positiver körperlicher Aktivitäten oder Erfahrungen. Problematisiert wird, dass trotz des Vorliegens von Körperbildstörungen die spezifischen Methoden seltener als erwartet auch tatsächlich zum Einsatz kamen. Dabei weisen die Evaluationen der Autoren darauf hin, dass eine Veränderung des Körperbilds mit dem Ausmaß des tatsächlichen Einsatzes körperbildbezogener Verfahren erfolgte.

Die Behandlungsbeschreibung von Stierle und Schult gibt ein anschauliches Beispiel für die kognitive Verhaltenstherapie bei einem Fall mit komorbid vorliegender körperdysmorpher Störung und Dermatillomanie. Das Kernmerkmal der körperdysmorphen Störung ist die exzessive Beschäftigung mit einem subjektiven Makel in der äußeren Erscheinung. Oftmals scheint ein Bereich besonders belastend zu sein, wie Merkmale des Gesichts oder des Kopfes. Dabei sollte aber nicht übersehen werden, dass oftmals sehr viele Körperbereiche als makelhaft erlebt werden und die Überzeugung der eigenen Entstelltheit sehr rigide von überwertiger Überzeugtheit bis zu wahnhaften Graden ausgeprägt sein kann (Kollei et al. 2012). Die Autoren zeigen sehr genau auf, dass gerade - die Veränderungsambivalenz berücksichtigend - akzeptanzfördernde Interventionen, wie das bewertungsfreie Wahrnehmen des gesamten Körpers bei Spiegelkonfrontation und Verhaltensexperimente zur Veränderung ungünstiger Einstellungen und Perfektionsansprüche therapeutischer Begleitung bedürfen und wiederholt durchgeführt werden sollten. Darüber hinaus ist es wichtig, die Bedeutung des Aussehens für den eigenen Selbstwert zu relativieren. In diesem Punkt weisen die körperbildbezogenen Therapien bei sowohl den psychischen Störungen als auch bei den sichtbaren Auffälligkeiten wie in den vorgenannten Beispielen starke Parallelen auf.
Körperbildbezogene Interventionen sind häufig Teil breiter angelegter Behandlungsansätze. Dabei belegen entsprechende Schwerpunktprogramme, dass sie Körperzufriedenheit und Verhaltenseinschränkungen verbessern. Therapien, die auf die Zielbereiche Einstellungen, Verhalten und Wahrnehmung (Jarry und Ip 2005) eingehen, zeigen insgesamt eine noch größere Wirksamkeit als Programme, die sich nur auf einzelne Teilbereiche konzentrieren. Dies betont noch einmal die Relevanz der Integration körperbildbezogener Therapien bei Personen mit aussehensbezogenen Ängsten und Einschränkungen.

\section{Korrespondenzadresse}

\section{Prof. Dr. A. Martin}

Klinische Psychologie und Psychotherapie

Bergische Universität Wuppertal

Gaußstr. 20, 42119 Wuppertal

martin@uni-wuppertal.de

Interessenkonflikt. Alexandra Martin und Jennifer Svaldi erklären, dass kein Interessenkonflikt besteht.

\section{Literatur}

Balantekin KN, Roemmich JN (2012) Children's coping after psychological stress. Choices among food, physical activity, and television. Appetite 59(2):298-304

Berger U, Schilke C, Strauss B (2005) Weight concerns and dieting among 8-12-year-old children. Psychother Psychosom Med Psychol 55:331-338

Jarry JL, Ip K (2005) The effectiveness of stand-alone cognitive-behavioral therapy for body image: a meta-analysis. Body Image 2:317-331

Kollei I, Brunhoeber S, Rauh E, de Zwaan M, Martin A (2012) Body image, emotions and thought control strategies in body dysmorphic disorder compared to eating disorders and healthy controls. J Psychosom Res 72:321-327

Naumann E, Tuschen-Caffier B, Voderholzer U, Lackner HK, Svaldi J (under review) Effects of acceptance and rumination on body dissatisfaction in eating disorders. J Abnorm Psychol

Roemmich JN, Wright SM, Epstein LH (2002) Dietary restraint and stress-induced snacking in youth. Obes Res 10(11):1120-1126

Roemmich JN, Lambiase MJ, Lobarinas CL, Balantekin KN (2011) Interactive effects of dietary restraint and adiposity on stress-induced eating and the food choice of children. Eat Behav 12(4):309-312

Stice E, Marti CN, Durant S (2011) Risk factors for onset of eating disorders: evidence of multiple risk pathways from an 8-year prospective study. Behav Res Ther 49(10):622-627

Wade T, George WM, Atkinson M (2009) A randomized controlled trial of brief interventions for body dissatisfaction. J Consult Clin Psychol 77(5):845-854 